\title{
Means and methods of noise protection to reduce the risk of cardiovascular disease in workers
}

\author{
Natalya Stuzhenko ${ }^{1}$, Mikhail Molev ${ }^{1}$, Irina Zanina ${ }^{1}$, Nataliya Kobzeva, ${ }^{2, *}$ \\ ${ }^{1}$ Don State Technical University, 1, pl. Gagarina, 344002, Rostov-on-Don, Russia \\ ${ }^{2}$ Rostov State Medical University, 29, Nakhichevan lane, 344022, Rostov-on-Don, Russia
}

\begin{abstract}
Some manufacturing processes are accompanied by significant noise and vibration. Sources of intense noise and vibration are machines and mechanisms with unbalanced rotating masses, as well as technological installations and apparatuses in which the movement of gases and liquids occurs at high speeds and has a pulsating character. The modern development of technology, equipping enterprises with powerful and fastmoving machines and mechanisms leads to the fact that people are constantly exposed to noise of increasing intensity. Increasing noise and vibration in the workplace has a harmful effect on the human body. As a result of prolonged exposure to noise, the normal activity of the cardiovascular and nervous systems, digestive and hematopoietic organs is disturbed, and professional hearing loss develops, the progression of which can lead to complete hearing loss.Among industrial hazards, noise and vibration occupy one of the leading places among production hazards. The harmful effects of increased noise levels on the human body are well khown, therefore, the relevance of this problem is obvious.
\end{abstract}

\section{Introduction}

When working in conditions of vibration, labor productivity decreases, the number of injuries increases. At some workplaces in agricultural production, vibrations exceed normalized values, and in some cases they are close to limit. The vibration levels on the controls do not always comply with the standards. Typically, low-frequency vibrations that negatively affect the body predominate in the vibration spectrum. Some types of vibration adversely affect the nervous and cardiovascular systems, the vestibular apparatus. The most harmful effect on the human body is provided by vibration, the frequency of which coincides with the frequency of natural vibrations of individual organs, the approximate values of which are as follows $(\mathrm{Hz})$ : stomach - $2 \ldots 3$; kidneys - 6 .. 8; heart - 4 ... 6; intestines - 2 ... 4; vestibular apparatus - 0.5..L, 3; eyes - 40 ... 100, etc.

The effect on muscular reflexes reaches $20 \mathrm{~Hz}$; the tractor-loaded seat on the tractor has its own vibration frequency of $1.5 \ldots 1.8 \mathrm{~Hz}$, and the rear wheels of the tractor - $4 \mathrm{~Hz}$. The vibration is transmitted to the human body at the moment of contact with the vibrating object: when acting on the limbs, local vibration occurs, and on the whole body - general.

\footnotetext{
*Corresponding author:5976765@mail.ru
} 
Local vibration affects neuromuscular tissues and the musculoskeletal system and leads to spasms of peripheral vessels. With prolonged and intense vibrations, in some cases, a professional pathology develops (local vibration often leads to it): peripheral, cerebral or cerebral-peripheral vibrational disease. In the latter case, there are changes in cardiac activity, general excitement, or, conversely, inhibition, fatigue, the appearance of pain, a feeling of shaking of the internal organs, nausea. In these cases, vibrations also affect the osteoarticular apparatus, muscles, peripheral circulation, vision, hearing [1,3,5,7,9]. Local vibrations cause spasms of blood vessels that develop from the terminal phalanges of the fingers, spreading to the entire hand, forearm, and cover the vessels of the heart.

Workers can be exposed to occupational vibration through the use of power or pneumatic hand tools or other machinery, or by driving large transportation, construction or agricultural vehicles. Vibration that is generated through the use of powered hand tools, and is transmitted from the tool to the hand-arm system is referred to as hand-transmitted vibration. However, recent studies have also demonstrated that vibration can be transmitted through platforms workers are standing on, and in these situations, the point of contact is the feet. Workers can also be exposed to whole body vibration (WBV). WBV exposure occurs in occupations where workers are driving trucks, large earth moving vehicles, or where they are using hand tools where the amplitude of the vibration is great enough to be transmitted to other portions of the body, such as in workers using jack-hammers.

Exposure to WBV is of concern within the workforce because it's associated with the development of a number of negative health consequences including back and neck pain, and potentially, cardiovascular disease, the development of various neuropathies, digestive problems, headaches, dizziness, motion sickness and possibly cancer. However, workers exposed to WBV are often also exposed to a number of other risk factors that may contribute to the development of these negative health effects. These risk factors include maintaining a static posture for a long-period of time, torque or twisting of the abdomen to view the area around the vehicle, and heavy lifting that often occurs when a vehicle is being loaded or unloaded. In addition to vibration and the physical exposures associated with a job, there may be other co-exposures to chemicals or certain environmental conditions that contribute to the development of disease or injury in workers. Because most workers are exposed to multiple factors that may induced injury or illness, it's difficult to determine which factors pose the greatest risk for inducing injury or illness. Experimental studies, examining the effects of each of these factor individually on health outcomes can provide additional information that will help determine the contribution of each exposure factor to various health problems.

This review will describe the industrial sectors where vibration exposure is most prevalent and the health effects associated with exposure to HTV and WBV. Experimental methods used to measure and characterize vibration generated in various occupational settings are discussed, and models that have been used to uncover the etiology of vibrationinduced injuries. Although numerous studies have been published on both HTV and HBV, there are new epidemiological studies showing an increased risk of specific cancers with exposure to WBV. Therefore additional etiological studies need to be performed. New avenues for research are discussed below.

The fight against noise is carried out by various methods and means:

- reduction of sound radiation power of machines and assemblies;

- localization of the action of sound by constructive and planning solutions;

- organizational and technical measures;

- treatment and prophylactic measures;

- the use of personal protective equipment for workers.

Conventionally, all means of protection against noise are divided into collective and individual.

Collective remedies: 
- means that reduce noise in the source;

- means that reduce noise along the path of its propagation to the protected object.

Noise reduction in the source of occurrence is the most effective and economical, (reduces noise by $5-10 \mathrm{~dB}$ ):

- elimination of gaps in gear joints;

- the use of globoid and chevron compounds as less noisy;

- widespread use, if possible, of plastic parts;

- elimination of noise in bearings;

- replacement of metal cases with plastic;

- balancing of parts (elimination of imbalance);

- elimination of misalignment in bearings;

- replacement of gears with V-belts;

- replacement of rolling bearings for sliding $(15 \mathrm{~dB})$, etc.

\section{Methods}

To reduce noise in the reinforcement workshops it is advisable: the use of hard plastics for coating surfaces in contact with the reinforcing wire; installation of elastic materials in places of falling reinforcement; the use of vibration-absorbing materials in the enclosing surfaces of machines.

Technological measures to reduce the noise level in the source include: reducing the amplitude of oscillations, speed, etc [1,3,5,7,9].

Means and methods of collective protection that reduce noise along the path of its distribution are divided into:

- architectural and planning;

- acoustic;

- organizational and technical.

\subsection{Architectural and planning measures to reduce noise}

From the point of view of noise control in urban planning, when designing cities, it is necessary to clearly divide the territory into zones: residential (residential), industrial, communal-warehouse and external transport, in accordance with the standards of sanitary protection zones when developing a general plan[11].

The correct layout of production facilities should be made taking into account the isolation of the room from external noise and noisy production. Industrial buildings with noisy technological processes should be placed on the leeward side in relation to other buildings and the residential village, and always with the end faces to them. \{The relative orientation of the buildings is decided so that the sides of the buildings with windows and doors are against the blind sides of the buildings. The window openings of such workshops are filled with glass blocks, and the entrance is made with tambours and a seal around the perimeter.

The most noisy and harmful production is recommended to be completed in separate complexes with ensuring gaps between individual nearby objects in accordance with sanitary standards. Indoors are also combined with noisy technologies, the number of workers exposed to noise is limited. Between buildings with noisy technology and other buildings of the enterprise it is necessary to observe gaps (at least $100 \mathrm{~m}$ ). The gaps between workshops with noisy technology and other buildings should be landscaped. Foliage of trees and shrubs serves as a good absorber of noise. New railway lines and stations should be separated from residential buildings by a protective zone with a width of at least $200 \mathrm{~m}$. When installing along the line of noise shields, the minimum width of the 
protective zone is $50 \mathrm{~m}$ (fig.1). Residential buildings should be located at least $100 \mathrm{~m}$ from the edge of the carriageway of express roads $[2,4,6,8]$.

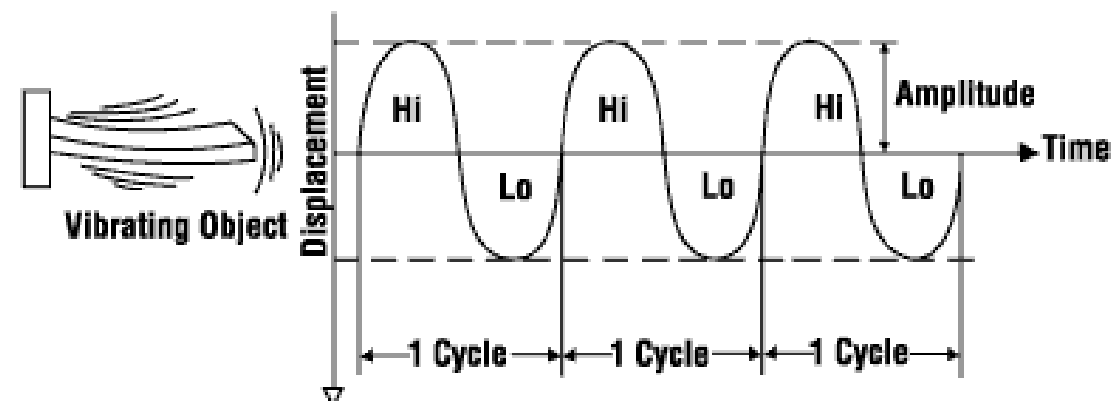

Fig. 1. Representation of the Measures of Vibration Exposure.

Noisy workshops should be concentrated in one or two places and separated from such premises by gaps or premises in which people are for a short time. In workshops with noisy equipment, you must correctly place the machines. They should be positioned so that increased noise levels are observed over a minimum area. Partitions are arranged between sections with different noise levels or utility rooms, warehouses for raw materials, finished products, etc. are placed. For enterprises located within the city, the noisiest rooms are located in the depths of the territory.

\subsection{Acoustic noise protection}

These include: sound insulation, sound absorption, sound suppression (noise suppression).

Sound insulation is the ability of structures that enclose or separate rooms, or their elements, to weaken the sound passing through them[10].

Types of sound insulation and sound insulation efficiency.

When sound energy meets the fence, part of it passes through the fence, part of it is reflected, part of it is converted into thermal energy, part of it is emitted by an oscillating barrier, and part of it is transformed into body sound that propagates inside the enclosure in the room.

The value of the emitted sound energy is much less than the sound energy acting on the fence from the side of the noise source, since part of the sound energy is reflected from the fence.

For most building structures and materials, there are tables with experimental data on their soundproofing ability in the active frequency band. When designing the fencing of buildings and structures, one of the criteria for choosing materials of walls, ceilings, partitions is their sound-insulating ability.

\subsection{Multilayer Soundproof Fencing}

To increase sound insulation and reduce the mass of fencing, multilayer fencing is used. For this, the space between the layers is filled with porous-fibrous materials and an air gap of 40-60 mm wide is left.

The lower the elasticity of the intermediate material, the lower the transmission of vibrations to the second cladding layer, and the higher the sound insulation (in practice, double fencing reduces the noise level by $60 \mathrm{~dB}$ ).

Sound absorption

In noisy rooms, the sound level increases significantly due to its reflection from building structures and equipment. The proportion of reflected sound can be reduced by 
applying special acoustic processing of the room, which consists in lining the internal surfaces with sound-absorbing materials.

To combat noise, suspended or piece sound absorbers, cubic or conical in shape, made of perforated plywood, plastic, metal filled with porous sound-absorbing m, are also used.

\section{Results}

Personal protective equipment against noise. In cases where technical means cannot reduce noise and vibration to acceptable limits, personal protective equipment is used. To reduce noise, SDS 3.3.6-037-99 recommends the use of personal protective equipment in accordance with GOST 12.1.003-88; for ultrasound (GOST 12.1.001-89). Personal protective equipment against noise should have the following basic properties:

- reduce noise to acceptable limits at all frequencies of the spectrum;

- Do not exert excessive pressure on the auricle;

- do not reduce the perception of speech;

- do not drown out the hazard sound signals;

- meet hygiene requirements.

Individual hearing protection includes internal and external anti-noise (antiphons), antinoise helmets.

The simplest of the internal anti-noise agents are cotton, gauze, sponge, etc., inserted into the auditory canal. Vata reduces noise by $3-14 \mathrm{~dB}$ in the frequency band from 100 to $6000 \mathrm{~Hz}$; cotton wool with wax - up to $30 \mathrm{~dB}$. Safety sleeves (ear plugs "Ear plugs") are used, which tightly close the auditory canal and reduce noise by $20 \mathrm{~dB}$.

External antinoise agents include antiphones that cover the auricle. Some anti-noise designs provide noise reduction of up to $30 \mathrm{~dB}$ at frequencies of the order of $50 \mathrm{~Hz}$ and up to $40 \mathrm{~dB}$ at frequencies of $2000 \mathrm{~Hz}$. Antiphons tire a person. Antiphons having selectivity, i.e. protecting hearing organs from the penetration of sound of undesirable frequency and transmitting sounds of a certain frequency. Recently, earphones anti-noise PSh-00, helmet anti-noise VTsNIIOT-2 are used. They are very effective in high-frequency noise, but keep in mind that they are not very convenient to use and can only be used temporarily. At a noise level of more than $120 \mathrm{~dB}$, headphones and earbuds do not provide the necessary noise attenuation.

\section{Discussion}

The influence of noise and vibration on a person and his body in recent decades has become one of the most urgent problems in all countries of the world. Noise affects a person in production (meaning industrial enterprises and some noise objects), the street and in the house.

From an unsatisfactory state of affairs with life safety, the country annually suffers large human, financial, economic, material and moral losses. Ensuring the safety of production and labor protection of workers is one of the main problems of the national security of the country. At present, in our country, many enterprises do not observe safety precautions, and working conditions cannot be called favorable.

The noise level on a hammer with a separate bed with an eccentric shock is $10 \neg-15$ dBA higher than with a central one, which is associated with the excitation of vibration of the racks by the beam through the guides. To exclude eccentric impacts, preliminary stamping operations must be performed on special equipment (for example, forging rollers), and gaskets made of pagulan type material with a thickness of $1 \mathrm{~mm}$, designed for pressure up to 10 , should be installed in the joints of guides with racks of the bed of the hammer. $\mathrm{kgf} / \mathrm{mm} 2$ and temperature up to $80 \mathrm{C}$. 
It is shown that the noise level from a hammer with a round bed profile and a structural damper built into the cavity of the bed stands is $10 \mathrm{dBA}$ lower than from a hammer with an open bed profile. The profile of the Shabot must be convex in order to avoid the formation of a reflected wave (fig.2).

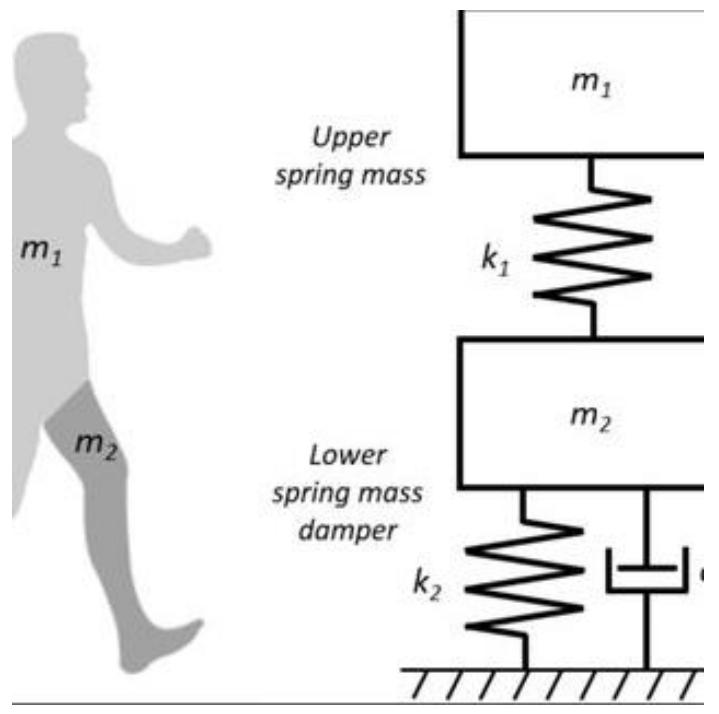

Fig. 2. Vibration effects.

The structural damping of the bed can be increased by closing the rack profile by attaching screens with a sound-absorbing coating (for example, from a layer of mineral wool) on it and installing partitions inside the closed cavities with filling the cells between the partitions with sand, gravel or shot.

To combat noise and vibration, both general and individual protective equipment is used. When planning production facilities, such as an engine test station, thermal and blacksmith shops are located on the leeward side in relation to other buildings and a residential area.

To attenuate the noise penetrating outward, it is necessary to use sound insulation of building envelopes. The rationalization of technological processes, the use of silencers, the careful fit of all moving parts of mechanisms - all this many times reduces noise. The greatest effect is achieved by replacing noisy jobs with less noisy ones.

Pneumatic riveting of frames and other parts must be replaced by hydraulic riveting or welding, forging and stamping by pressing.

To reduce vibration, it is also necessary to use special sound-absorbing structures near sources of noise or a workplace, enclose noisy units of the unit in insulating covers (gear reducers, chain, belt and other gears, impact parts, engines). When processing metal rods on automatic turret machines, the safety tube must be spring loaded or a rubber jacket inserted in the hole of this tube.

In order to reduce vibration, it is recommended to use a stiff, spring-free seat in cars, as it is a good vibration damper. Vibration acts on a person through the back, pelvis, arms. Long-term operation of the car leads to fluctuations in body parts, which negatively affects the driver. To reduce vibration, machines should be installed on a foundation deepened below the foundation of the walls, insulated from the soil by air gaps, or on specially designed shock absorbers made of steel springs or made of elastic materials.

To weaken the transmission of vibrations and noise through air ducts and pipelines, they must be connected to the fans and pumps using a flexible insert made of rubberized fabric or a rubber pipe. 
It is necessary to cover vibrating surfaces and equipment with vibration absorbing and damping materials (rubber, special mastics, asbestos, bitumen, Agate plastics, etc.). At the connection points of the mating parts, shock absorbing materials (rubber, cork, cardboard, asbestos, spring shock absorbers) should be used to ensure a snug fit.

Reduce vibration in the vibration source, i.e. in the source of its formation, the following methods are possible: by excluding the impact interaction of the parts from the structure, by replacing the reciprocating movement of the parts by the rotational one, by eliminating the imbalance of rotating parts and machine components.

When working with pneumatic and electric manual machines, vibration occurs, transmitted through the handles and cases to the hands of workers, and sometimes to the legs through the medium being processed, usually when working with rammers and vibrators. To reduce vibration in this case, use handles with vibration damping or automation devices.

Personal protective equipment against vibration is used when other means are ineffective.

Shoes with shock-absorbing soles, handguards with vibration-absorbing elastic gaskets, etc. are used as personal protective equipment against vibration.

Under the influence of intense noise and vibration, fatigue and irritability, poor sleep, headache, weakening of memory, attention and visual acuity occur, which leads to a decrease in labor productivity (on average by 10-15\%) and is often the cause of injuries. Vibration and noise affect the cardiovascular, endocrine and nervous systems, disrupt the coordination of movements. Adaptation to noise is not possible.

\section{Acknowledgements}

The study had no sponsorship.

\section{References}

1. A.T. Rybak, V.P. Zharov, A.V. Serdyukov, Russian Engineering Research 29(2), 194197 (2009)

2. A. Rybak, O. Lyakhnitskaya, MATEC Web of Conferences 132, 01001 (2017)

3. A.T. Rybak, A.R. Temirkanov, O.V. Lyakhnitskaya, Russian Engineering Research 38(9), $702-704$ (2018)

4. A.T. Rybak, A.R. Temirkanov, O.V. Lyakhnitskaya, Russian Engineering Research 38(3), 212-217 (2018)

5. K. Kobzev, S. Shamshura, A. Chukarin, V. Bogdanovich, V. Kasyanov, MATEC Web of Conferences 226, 01022 (2018)

6. K. Kobzev, S. Shamshura, A. Chukarin, A. Buryanovand, V. Kasyanov, MATEC Web of Conferences 226, 01023 (2018)

7. K. Kobzev, A. Chukarin, IOP Conference Series: Earth and Environmental Science 403, 012145 (2019)

8. K.O. Kobzev, E.S. Bozhko, A.V. Mozgovoi, M.D. Molev, N.I. Stuzhenko, IOP Conference Series: Materials Science and Engineering 680, 012014 (2019)

9. K.O. Kobzev, E.S. Bozhko, A.V. Mozgovoi, E.I. Kostromina, L.G. Babenko, IOP Conference Series: Materials Science and Engineering 680, 012013 (2019)

10. N. Kobzeva, V. Terentev, I. Zolotuhina, IOP Conference Series: Materials Science and Engineering 680, 012046 (2019) 
11. N. Kobzeva, V. Terentev, I. Zolotuhina, IOP Conference Series: Materials Science and Engineering 680, 012011 (2019) 